\title{
LIPOSA pharmacopuncture, a new herbal formula, affects localized adiposity by regulating lipid metabolism in vivo
}

\author{
HAESU LEE ${ }^{1,2}$, MI HYE KIM ${ }^{1}$, SEONG CHUL JIN ${ }^{1}$, LA YOON CHOI ${ }^{1}$, \\ YEON KYUNG NAM ${ }^{1}$ and WOONG MO YANG ${ }^{1,2}$ \\ ${ }^{1}$ Department of Convergence Korean Medical Science, College of Korean Medicine, Kyung Hee \\ University, Seoul 02447; ${ }^{2}$ Boin Bio Convergence Co., Ltd., Seoul 02455, Republic of Korea
}

Received August 27, 2020; Accepted December 3, 2020

DOI: $10.3892 /$ etm.2021.10725

\begin{abstract}
Localized adiposity is a serious aesthetic problem and a well-known health risk factor. There is a growing interest in minimally invasive treatment options for excessive fat accumulation, such as pharmacopuncture. LIPOSA is a newly developed pharmacopuncture formula from three natural herbs: The tuber of Pinellia ternata (Thunb.) Breitenb., the whole plant of Taraxacum platycarpum Dahlst. and the root of Astragalus membranaceus Bunge. The present study investigated the effects of pharmacopuncture treatment with LIPOSA on localized adiposity. Male C57BL/6J mice were fed high fat diet for 8 weeks to induce obesity. Then, $100 \mu 1$ LIPOSA was injected into the left-side inguinal fat pad at various concentrations, including 13.35, 26.7 and $53.4 \mathrm{mg} / \mathrm{ml}$. Normal saline was injected into the right-side inguinal fat pad of each mouse as a control. The treatment was performed three times per week for 2 weeks. The weight and histological changes were analyzed in the inguinal fat pad of the obese mice. The expression levels of adipose triglyceride lipase (ATGL), hormone-sensitive lipase (HSL), autophagy-related gene (ATG)5, ATG7 and LC3-II, as lipophagy-related factors, were evaluated to confirm the lipid-catabolic effects of LIPOSA. LIPOSA pharmacopuncture markedly decreased the weight of the fat tissue and the size of the adipocytes in the inguinal region of the mouse models of obesity in a dose-dependent manner. The expression levels of ATGL, HSL, ATG5, ATG7 and LC3-II were significantly increased by the LIPOSA treatments. In addition, LIPOSA pharmacopuncture was found to decrease the expression levels of ACC, PPAR- $\gamma$ and PEPCK. The results indicated that subcutaneous injection of LIPOSA can degrade local fat and induce lipophagic and
\end{abstract}

Correspondence to: Professor Woong Mo Yang, Department of Convergence Korean Medical Science, College of Korean Medicine, Kyung Hee University, 26 Kyungheedae-ro, Dongdaemun-gu, Seoul 02447, Republic of Korea

E-mail:wmyang@khu.ac.kr

Key words: LIPOSA, pharmacopuncture, local fat, adiposity, lipid droplet, lipolysis lipase activation effects. In addition, lipid metabolism related to fat accumulation was regulated by the LIPOSA treatment. The present study suggests that LIPOSA pharmacopuncture can be a non-surgical alternative in the treatment of localized adiposity.

\section{Introduction}

Excessive fat accumulation in obesity is a serious aesthetic problem (1). The regional distribution of local fat is a well-known risk factor of obesity rather than body mass index (BMI) (2). Abdominal adiposity, with subcutaneous or visceral fat deposition, is implicated in several medical conditions such as metabolic syndrome, cardiovascular disease and lower quality of life (3). Aesthetic treatment for localized adiposity is performed mainly for abdominal subcutaneous adipose tissue (4). Liposuction, suction-assisted lipectomy, is the most commonly used technique of plastic surgery procedures in North America (5). Although fat tissue can be extracted by liposuction effectively, complications may cause contour deformities, embolism and even death (5). Injections of phosphatidylcholine (PC) and sodium deoxycholate (DC) have been widely used as a minimally invasive treatment for localized adiposity (6). However, PC and DC injection may have substantial side effects including fibrosis and necrosis of the tissue (7). Pharmacopuncture, a new acupuncture technique with the injection of a herbs extract at the acupuncture point, could be a non-surgical alternative in the treatment of localized adiposity (8).

LIPOSA, consisting of the tuber of Pinellia ternata (Thunb.) Breitenb., the whole plant of Taraxacum platycarpum Dahlst. and the root of Astragalus membranaceus Bunge, is a newly developed formula of pharmacopuncture treatment for localized adiposity. P. ternata, T. platycarpum and A. membranaceus have been used for treating metabolic disorders including obesity as traditional Korean medicines (9-11). In the theories of traditional Korean medicine, obesity can be caused by 'phlegm dampness', 'deficiency of Qi' and 'pathologic dampness-heat' (12). P. ternata and T. platycarpum are known to be effective herbs for dispelling 'phlegm dampness' and 'dampness-heat', respectively (12). A. membranaceus is one of the most frequently prescribed 'Qi tonifying' herbs in traditional Korean medicine (12). 
P. ternata, T. platycarpum and A. membranaceus can be used for the treatment of obesity, however, the effects of pharmacopuncture with these herbs on localized adiposity have not been studied yet. In this study, we investigated the efficacy of LIPOSA pharmacopuncture on localized adiposity by analyzing the fat pad weight and histological changes of the fat tissues in obese mice. To confirm the underlying mechanism of then LIPOSA pharmacopuncture on the inhibition of local fat, lipolytic enzymes including adipose triglyceride lipase (ATGL) and hormone-sensitive lipase (HSL) and lipophagic molecules including LC3-II, autophagy-related gene (ATG) 5 and ATG7 were investigated in high fat diet (HFD)-induced obese mice. Moreover, lipogenesis-related factors such as acetyl-CoA carboxylase (ACC) and phosphoenolpyruvate carboxykinase (PEPCK) and peroxisome proliferator-activated receptors (PPAR)- $\gamma$ as an adipogenetic biomarker were evaluated in the inguinal fat tissues of the obese mice.

\section{Materials and methods}

Preparation of LIPOSA pharmacopuncture. LIPOSA pharmacopuncture compose A. membranaceus, T. platycarpum and P. ternata. A. membranaceus and T. platycarpum were extracted with 20 -folds of distilled water at $100^{\circ} \mathrm{C}$ for $2 \mathrm{~h}$ by refluxing. $P$. ternata was extracted with 15 -folds of distilled water at RT for $2 \mathrm{~h}$. Each extract was filtered by $3 \mu \mathrm{m}$ paper filers, respectively, and then mixed. Mixed extracts were evaporated and freeze-dried. The yields of mixed extracts were $8.65 \%$. Dried extracts were diluted in normal saline and compensated the $\mathrm{pH}$ range from 6.8 to 7.2 by $1 \mathrm{~N} \mathrm{NaOH}$ solution.

Animal treatment. Male C57BL/6J mice (5 weeks old) were purchased from Raonbio Inc. Mice were housed under temperature- and humidity-controlled facility. After 1 week of housing, all mice were fed high-fat diet (HFD) containing $60 \%$ fat for 8 weeks to induce obesity. To monitor and compare any adverse effects of LIPOSA pharmacopuncture with normal, 8 normal mice were fed standard diet. Body weight was measured once a week until the end of the animal experiments. Following 8 weeks of HFD, mice $(n=8)$ were divided into 3 groups in accordance with the weight of the mice, which was LIPOSA 13.35, 26.7, $53.4 \mathrm{mg} / \mathrm{ml}$. We conducted previous experiments to determine the effective doses of LIPOSA. Effective dose ranges of Pinellia ternate, Taraxacum platycarpum and Astragalus membranaceus were 5-10, 2-4 and 15-20 mg/ml, respectively. Therefore, a tentative dose $(26.7 \mathrm{mg} / \mathrm{ml})$ with half dose $(13.35 \mathrm{mg} / \mathrm{ml})$ and double dose $(53.4 \mathrm{mg} / \mathrm{ml})$ of LIPOSA were tested to establish the actual therapeutic dose of LIPOSA in this study. Obese mice were used as self-control, vehicle (normal saline) was injected in the right inguinal fat pad and LIPOSA were injected in the left inguinal fat pad. The samples were injected $100 \mu \mathrm{l}$ each, 3 times a week for 2 weeks. Body weight and food intake were monitored every week. No significance was observed in body weight of LIPOSA-treated groups, suggesting that LIPOSA had inhibitory effects against localized fat accumulation rather than body weight reduction (Fig. S1). During the treatment of LIPOSA, no significant differences of daily food intake were shown in LIPOSA-treated mice (Table SI). In addition, there was no sign of toxicity in all LIPOSA-treated mice. All animal procedures were approved by Committee on Care and Use of Laboratory Animals of the Kyung Hee University (KHUASP(SE)-18-070; Seoul, Korea).

Measurement of inguinal fat weight. At the end of the 10 weeks, all animals under anesthesia were scanned from total-body scanner (InAlyzer dual X-ray absorptiometry; Medikors). Dual energy X-ray absorptiometry (DXA) measures one time with low energy and one time with high energy to separate the images into tissues in gram units by separating them into fat and lean before analysis. Fat distribution mice was visualized by body composition view by a mapping image processed by a software in the device. Red, blue and white color indicates the fat tissue, lean tissue and bone tissue, respectively. After then, all mice were sacrificed under anesthesia with $1 \%$ avertin (cat. no. T4,840-2; Sigma-Aldrich; Merck KGaA). Blood samples were collected by orbital puncture. Mice are euthanized by cervical dislocation. Inguinal fat pad was collected from the thigh and weight was measured. The regions to determine the weight of inguinal fat pad were from knee to tail based on line of ventral spine. The inguinal fat pad weight by LIPOSA treatment was calculated by relative intensity that the saline-treated fat weight was converted to 1 .

Histology. After 10 weeks of feeding experimental diets, inguinal fat pad was collected from the C57BL/6 mice. Inguinal fat tissues were fixed in $10 \%$ neutralized formalin. The dehydrated fat tissue was then embedded in paraffin wax. Histological sections of $5 \mu \mathrm{m}$ thickness were stained with hematoxylin and eosin (H\&E). Adipocyte size was evaluated in 6 mice from each group and 6 random fields (magnification, $\mathrm{x} 400$ ) per mice. To measure cross-sectional adipocyte area, micrographs were taken using a light microscope (Nikon) and analyzed by using the ImageJ software.

Western blotanalysis. Proteins were extracted by homogenizing inguinal fat tissues in the tissue protein extraction reagent (T-PER) including protease inhibitor cocktail. The homogenate was extracted in ice for $2 \mathrm{~h}$ and centrifuged at 17,000 rpm for $30 \mathrm{~min}$. Supernatant was collected and quantified using Bradford assay. $20 \mu \mathrm{g}$ of protein samples were electrophoresed in $10 \%$ SDS-PAGE gels for $2 \mathrm{~h}$ at $100 \mathrm{~V}$. Proteins were transferred onto methanol-activated polyvinylidene difluoride (PVDF) membrane using trans-blot turbo transfer system (Bio-Rad Laboratories, Inc.). The membranes were blocked with 3\% BSA and washed with TBS-T buffer for 3 times. The membranes were blotted with primary antibodies $(1: 1,000$ dilution) for overnight at $4{ }^{\circ} \mathrm{C}$ and HRP conjugated secondary antibodies (1:3,000 dilution) were incubated for $1 \mathrm{~h}$ at RT. The target protein bands were detected by chemi-imaging system, Davinch-Chemi ${ }^{\mathrm{TM}}$.

Measurement of serum toxicity. Blood samples were separated by centrifuging the blood at 17,000 rpm for $20 \mathrm{~min}$ to determine the serum toxicity by enzyme-linked immunsorbent assay (ELISA). Serum biochemical indicators including blood urea nitrogen (BUN), creatinine, aspartate transaminase (AST) and alanine transaminase (ALT) were analyzed by Mouse Blood Urea Nitrogen ELISA kit (cat. no. MBS2611085; Mybiosource), 
Mouse Serum Creatinine ELISA kit (cat. no. MBS3807501; Mybiosource). Mouse Aspartate Aminotransferase ELISA kit (cat. no. MBS450720; Mybiosource) and Mouse Alanine Aminotransferase ELISA kit (cat. no. MBS264717; Mybiosource), respectively. Based on standard curve, serum BUN, creatinine, AST and ALT was calculated.

Statistical analysis. Significance between vehicle and LIPOSA was determined by paired Student's t-test. Serum BUN, creatinine, AST and ALT measurements and body weight differences were analyzed using one-way ANOVA and Tukey's multiple comparisons test. In all analyses, $\mathrm{P}<0.05$ was considered to indicate a statistically significant difference.

\section{Results}

Effects of LIPOSA pharmacopuncture on the inguinal fat weight in obese mice. The left inguinal fat administered with LIPOSA was significantly reduced compared to the vehicle side (Fig. 1A). Radiography of fat displayed by red color showed that LIPOSA treatment remarkedly decreased the fat deposition in inguinal fat pad (Fig. 1B). LIPOSA-treated groups exhibited decreases of inguinal fat weight ratio. LIPOSA treatments with $13.35,26.7$ and $53.4 \mathrm{mg} / \mathrm{ml}$ concentrations reduced the inguinal fat pad weight by $14.5,21.2$ and $19.0 \%$ in HFD-induced obese mice, respectively (Fig. 1C). Based on the DXA scan to measure the exact weight of inguinal fat, the ratio of fat weight administered with LIPOSA were significantly lower than that with vehicle. Subcutaneous injection with 13.35 , 26.7 and $53.4 \mathrm{mg} / \mathrm{ml}$ of LIPOSA significantly attenuated the inguinal fat pads weight by $26.2,19.6$, and $21.8 \%$, respectively (Fig. 1D).

Effects of LIPOSA pharmacopuncture on histological changes of inguinal fat tissues in obese mice. As shown in H\&E staining, fat diameter of in inguinal fat pad was markedly reduced by LIPOSA treatment compared to the vehicle side-fat pad (Fig. 2A). LIPOSA pharmacopuncture injection dose-dependently decreased the inguinal fat adipocyte size about $48.5,50.9$ and $61.4 \%$ (Fig. 2B and C).

Effects of LIPOSA pharmacopuncture on expressions of lipophagy-related factors in inguinal fat tissues in obese mice. The protein levels of LC3-II were significantly increased by about 2.9-, 2.8- and 4.1-fold in the LIPOSA 13.35, 26.7 and $53.4 \mathrm{mg} / \mathrm{ml}$-treated fat tissues compared to self-control, right side. Compared with saline-injected right side, the expressions of ATG5 were markedly upregulated to 3.1-, 3.0- and 4.6-folds in all three doses of LIPOSA group. Also, the ATG7 expressions were significantly increased by about 7.5-, 8.9- and 10.3-folds in the 13.35, 26.7 and $53.4 \mathrm{mg} / \mathrm{ml}$ of LIPOSA-treated fat tissues (Fig. 3).

Effects of LIPOSA pharmacopuncture on the expressions of lipolytic enzymes in inguinal fat tissues in obese mice. The levels of phosphorylated ATGL were increased about 1.5-, 1.8- and 1.5-folds in the inguinal fat tissues with LIPOSA $13.35 \mathrm{mg} / \mathrm{ml}$, LIPOSA $26.7 \mathrm{mg} / \mathrm{ml}$ and LIPOSA $53.4 \mathrm{mg} / \mathrm{ml}$ compared to vehicle side-fat tissues. In similar, compared with right side, the levels of phosphorylated HSL were elevated about 2.1-, 2.6- and 2.6-folds in the LIPOSA $13.35 \mathrm{mg} / \mathrm{ml}$, LIPOSA $26.7 \mathrm{mg} / \mathrm{ml}$ and LIPOSA $53.4 \mathrm{mg} / \mathrm{ml}$-treated inguinal fat tissues (Fig. 4).

Effects of LIPOSA pharmacopuncture on the expressions of ACC, PEPCK and PPAR- $\gamma$ in inguinal fat tissues in obese mice. In Fig. 5, the expression of phosphorylated ACC was significantly downregulated $69.5,70.0$ and $66.1 \%$, respectively, in $13.35,26.7$ and $53.4 \mathrm{mg} / \mathrm{ml}$ of LIPOSA-treated left inguinal fat pads. In addition, the levels of PEPCK were remarkedly attenuated by $27.4,21.3$ and $54.8 \%$, respectively, in the LIPOSA 13.35, LIPOSA 26.7 and LIPOSA 53.4 groups. PPAR- $\gamma$ expressions were lowly expressed by $38.7,53.3$ and $61.6 \%$ compared to right inguinal fat pads in dose-dependent manner.

Effects of LIPOSA pharmacopuncture on serum toxicity in obese mice. We evaluated levels of injury marker of liver and kidney to assess potential toxic effect of LIPOSA (Table I). The levels of BUN were $25.80 \pm 2.17,30.60 \pm 4.67$ and $29.00 \pm 2.92$ by LIPOSA injection. Creatinine levels in serum were $0.27 \pm 0.05$, $0.25 \pm 0.04$ and $0.29 \pm 0.03$ by injection of LIPOSA $13.35,26.7$ and $53.4 \mathrm{mg} / \mathrm{ml}$, respectively. The serum levels of AST were $187.50 \pm 40.83,230.75 \pm 20.56,173.25 \pm 44.30$ and ALT were $33.00 \pm 7.66,33.00 \pm 4.97,29.00 \pm 5.23$ in each LIPOSA treated groups. There was no significant difference of serum BUN, creatinine, AST and ALT levels in all three LIPOSA-treated groups. We conducted the additional evaluation of serum BUN, creatinine, AST (GOT) and ALT (GPT) in normal mice. Also, the levels of blood urea nitrogen (BUN), creatinine, AST and ALT were not changed by LIPOSA injection within normal range. We suggested that no toxicities in the serum levels of BUN, creatinine, AST and ALT by LIPOSA 13.35, 26.7 and $53.4 \mathrm{mg} / \mathrm{ml}$ injection.

\section{Discussion}

Lipolytic stimulation of fat cells by lipolytic injection or liposuction has been known to reduce localized body fat, that is intended to slim down specific body parts (13). Adipose cells, known as adipocytes, are the cells of adipose tissues that synthesize, store and release fat into the blood (14). In the condition of obesity by excessive fat intake, the size of adipocytes is about 10 times bigger than the original size (15). Enlargement of adipocytes in specific regions forms 'love handles' known as fat deposits (16). In this study, the potential of LIPOSA pharmacopuncture as a localized lipolytic material was investigated by comparing sample-injected left side and saline-injected right side for self-control. Subcutaneous injection with LIPOSA pharmacopuncture decreased the accumulation of inguinal fat tissues. As shown in the x-ray images, the red-indicated fat deposition was remarkedly reduced in the LIPOSA-treated site compared to the saline-treated site. In addition, the diameter of the adipocytes in the inguinal fat tissues was significantly decreased by the LIPOSA injection at all concentrations. Those results suggested that LIPOSA pharmacopuncture has a role as a lipolytic material in localized fat depositions.

'Lipolysis signaling' is defined as the hydrolysis of triacylglycerols stored in lipid droplets in the fat. There are 
A LIPOSA 13.35

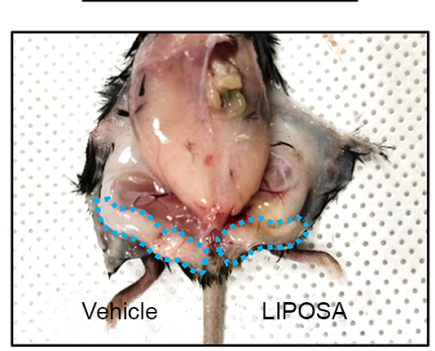

B

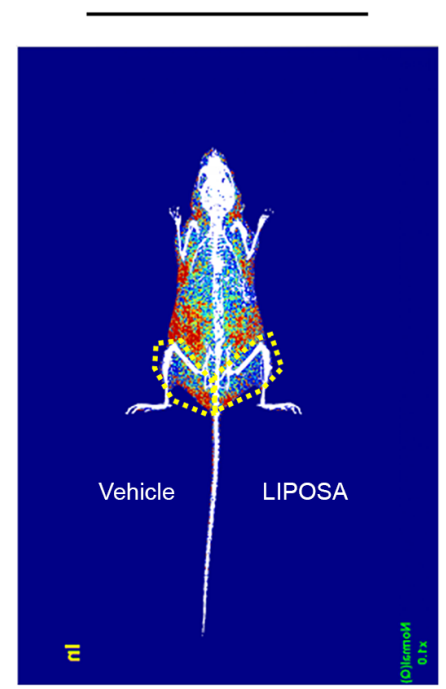

LIPOSA 26.7

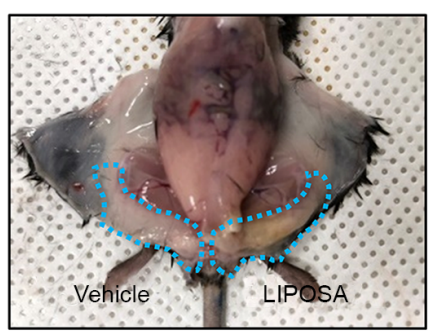

LIPOSA 26.7

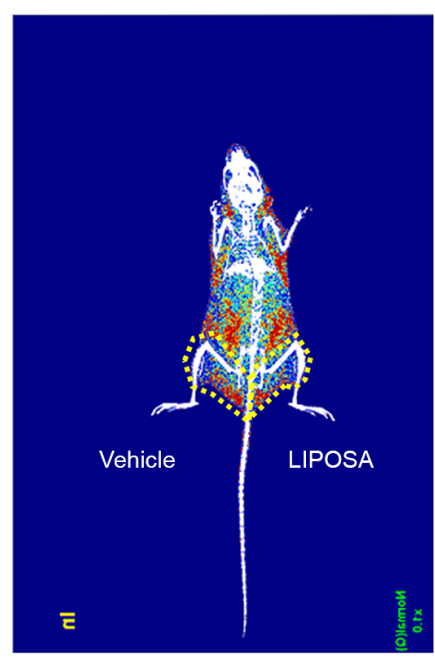

LIPOSA 53.4

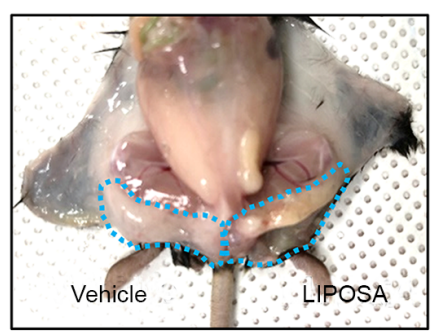

LIPOSA 53.4

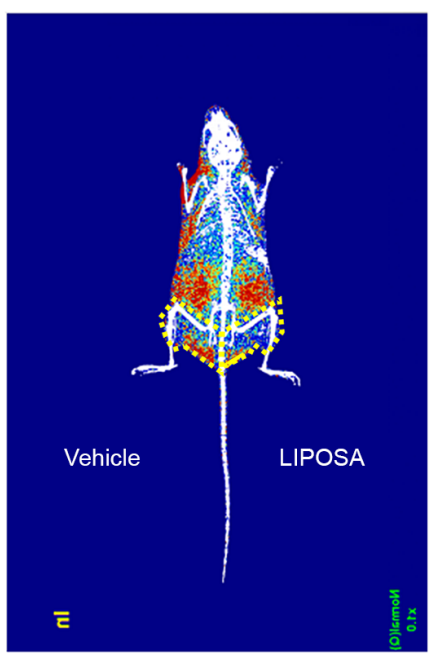

C

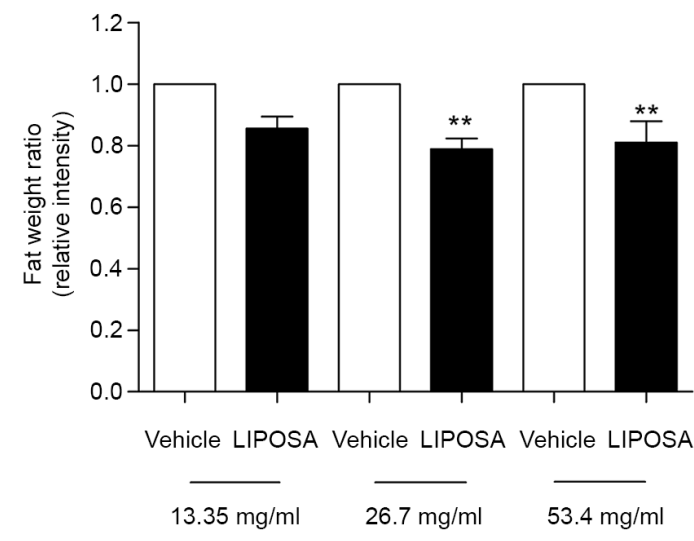

D

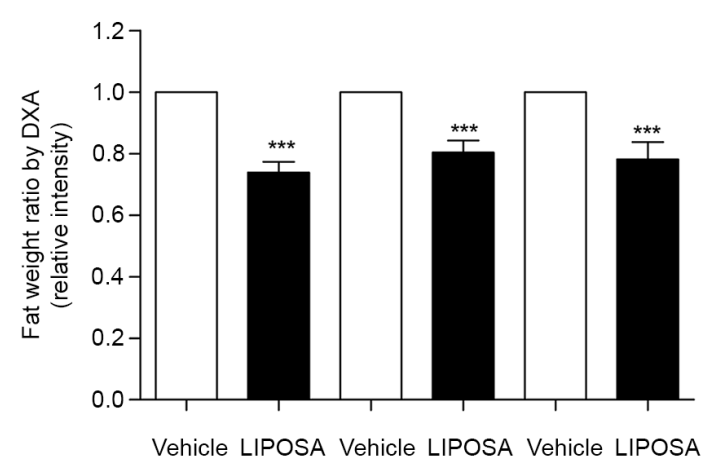

$13.35 \mathrm{mg} / \mathrm{ml}$

$26.7 \mathrm{mg} / \mathrm{ml}$

$53.4 \mathrm{mg} / \mathrm{ml}$

Figure 1. LIPOSA pharmacopuncture reduces the inguinal fat ratio. (A) Representative morphological images of inguinal fat tissues. Blue dots from knee to tail indicate the designated regions of inguinal fat. (B) Representative mapping images of body composition of mice constructed using DXA software. Yellow dots from knee to tail indicate the designated regions of inguinal fat. Red, fat tissue; blue, lean tissue; white, bone tissue. (C) Relative weight ratio of inguinal fat collected from knee to tail based on line of ventral spine at the end of the experiment. Relative ratio of fat weight after LIPOSA treatment was calculated when the saline-treated fat weight was normalized to 1. (D) Relative ratio of fat weight measured by targeting regions of interest (the designated regions of inguinal fat pad) indicated by yellow dots in DXA software. The saline-treated fat weight was normalized to 1 and the inguinal fat pad weight after LIPOSA treatment was calculated as the relative intensity. Quantitative data are presented as the mean \pm standard error of the mean. ${ }^{* *} \mathrm{P}<0.01$ and ${ }^{* * *} \mathrm{P}<0.001$ compared with each saline-treated side (vehicle).

several potential mechanisms related to lipolytic stimulation, which induces fat cell destruction (17). The neutral lipids in a lipid droplet breakdown into free fatty acids by lysosomal degradation autophagy, called lipophagy (18). The events of the lipophagic process are coordinated by components of the autophagic machinery, ATGs, with the generation of
LC3-II. ATG7 has been reported to regulate ATG5, leading to the conjugation of LC3 to a lipid. LC3-II activation in the lipid droplet forms the autophagosomes following the degradation of lipid stores (19). LC3 is reported to regulate ATGL-mediated lipid mobilization, although the contribution of lipases and lipophagy to lipolytic process has not 
A
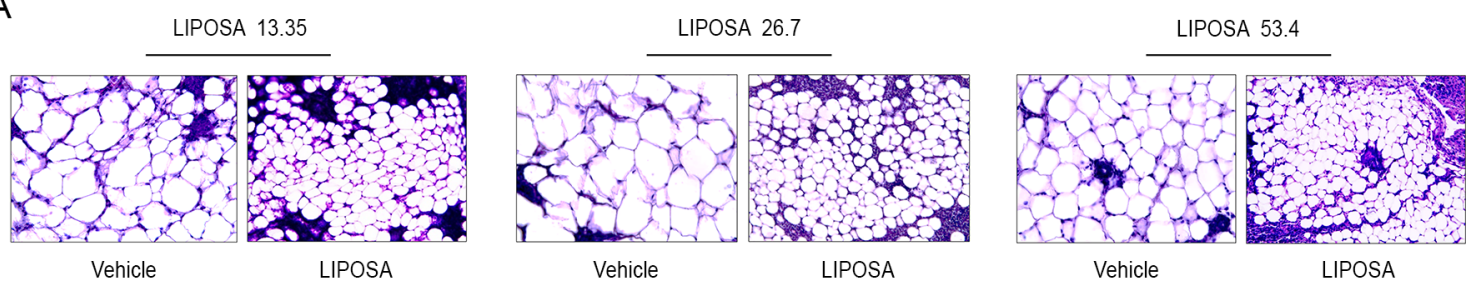

B

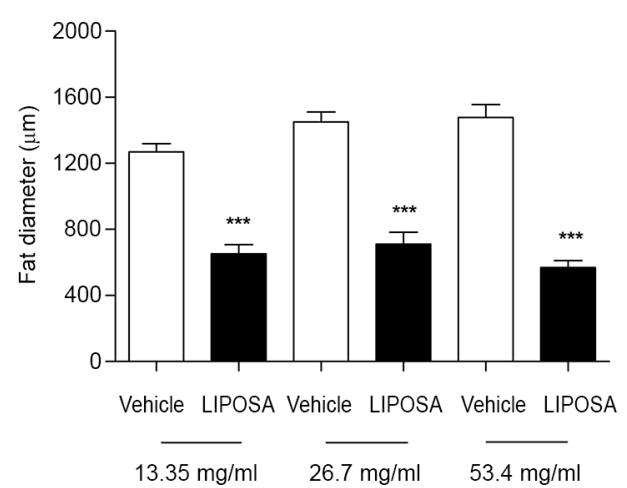

C

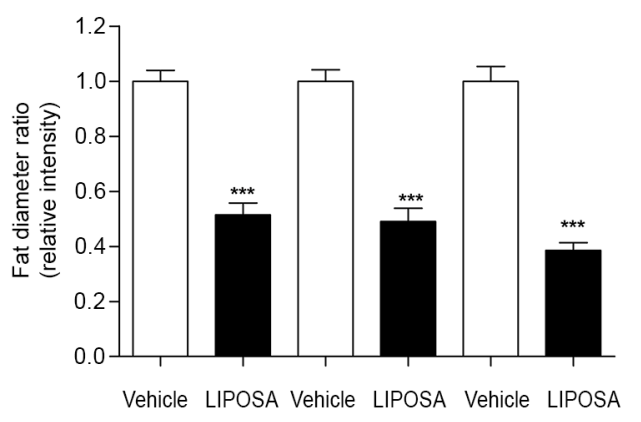

$\overline{13.35 \mathrm{mg} / \mathrm{ml}} \quad \overline{26.7 \mathrm{mg} / \mathrm{ml}} \quad \overline{53.4 \mathrm{mg} / \mathrm{ml}}$

Figure 2. LIPOSA pharmacopuncture decreases the fat diameter of inguinal fat tissues. (A) Representative histological images of inguinal fat tissues. Sections were stained by $\mathrm{H} \& \mathrm{E}$ and viewed under a microscope (magnification, $\mathrm{x} 400$ ). (B) Value of fat diameter in inguinal fat quantified using ImageJ software. (C) Relative value of fat diameter in inguinal fat measured compared with vehicle. The fat diameter in the saline-treated side was normalized to 1 and that in the LIPOSA-treated side was calculated as the relative intensity. Quantitative data are shown as the mean \pm standard error of the mean. ${ }^{* * *} \mathrm{P}<0.001$ compared with each saline-treated side (vehicle). $\mathrm{H} \& \mathrm{E}$, hematoxylin and eosin.
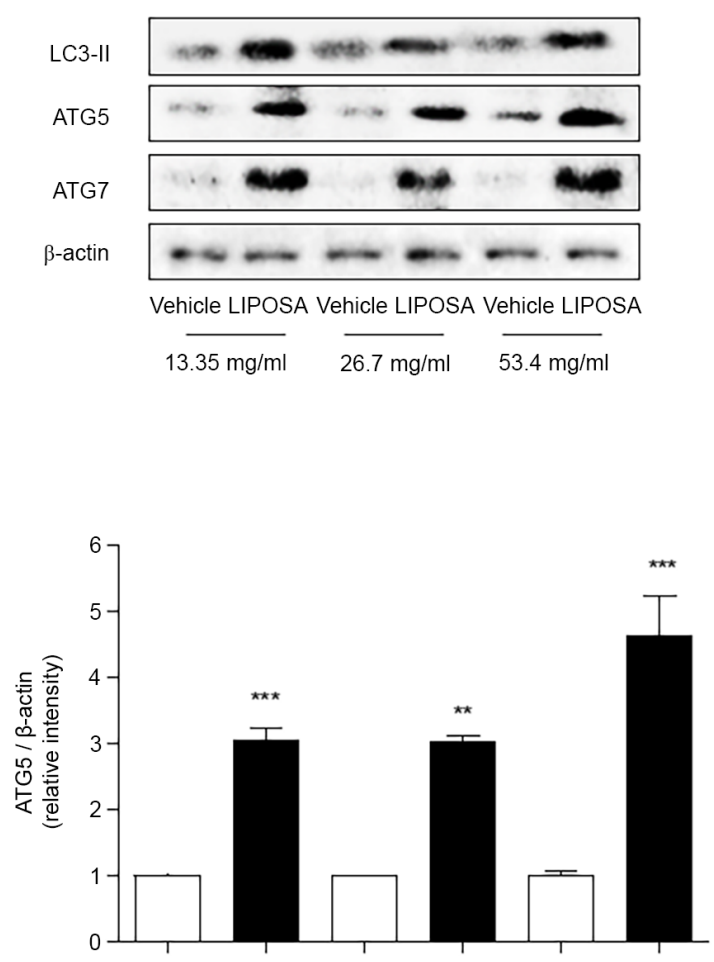

Vehicle LIPOSA Vehicle LIPOSA Vehicle LIPOSA

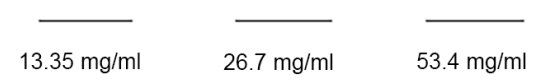

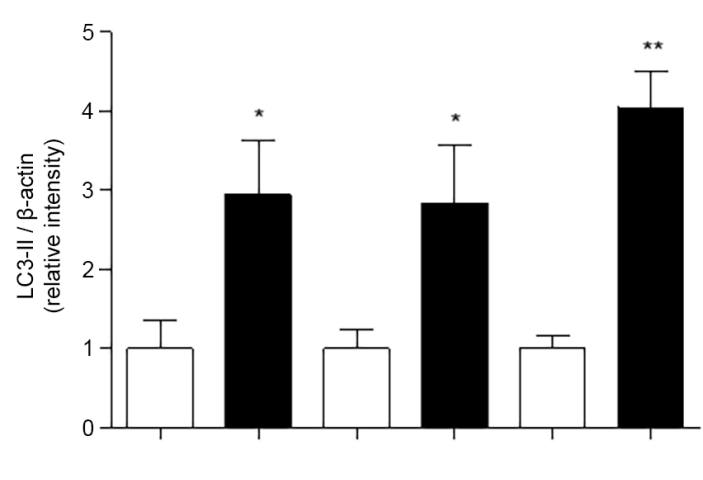

Vehicle LIPOSA Vehicle LIPOSA Vehicle LIPOSA

$13.35 \mathrm{mg} / \mathrm{ml} \quad 26.7 \mathrm{mg} / \mathrm{ml} \quad 53.4 \mathrm{mg} / \mathrm{ml}$

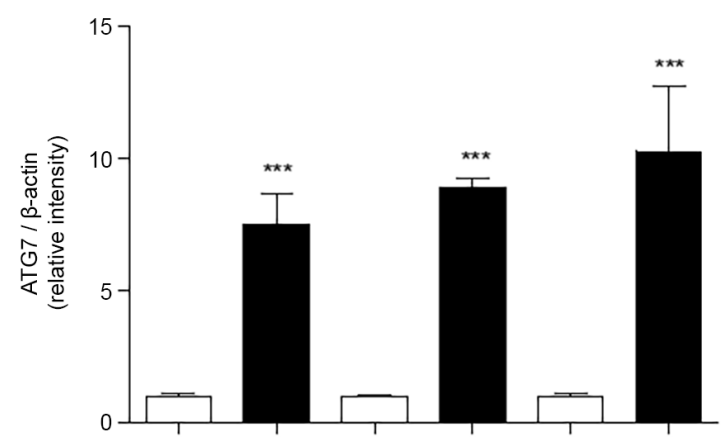

Vehicle LIPOSA Vehicle LIPOSA Vehicle LIPOSA

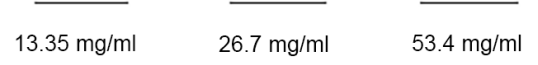

Figure 3. LIPOSA pharmacopuncture increases the expression of lipophagy-related factors in inguinal fat tissues in obese mice. Expression levels of LC3-II, ATG5 and ATG7 were analyzed by western blotting. Quantitative data are shown as the mean \pm standard error of the mean. ${ }^{*} \mathrm{P}<0.05,{ }^{* *} \mathrm{P}<0.01$ and ${ }^{* * * *} \mathrm{P}<0.001$ compared with each saline-treated side (vehicle). ATG, autophagy-related gene. 

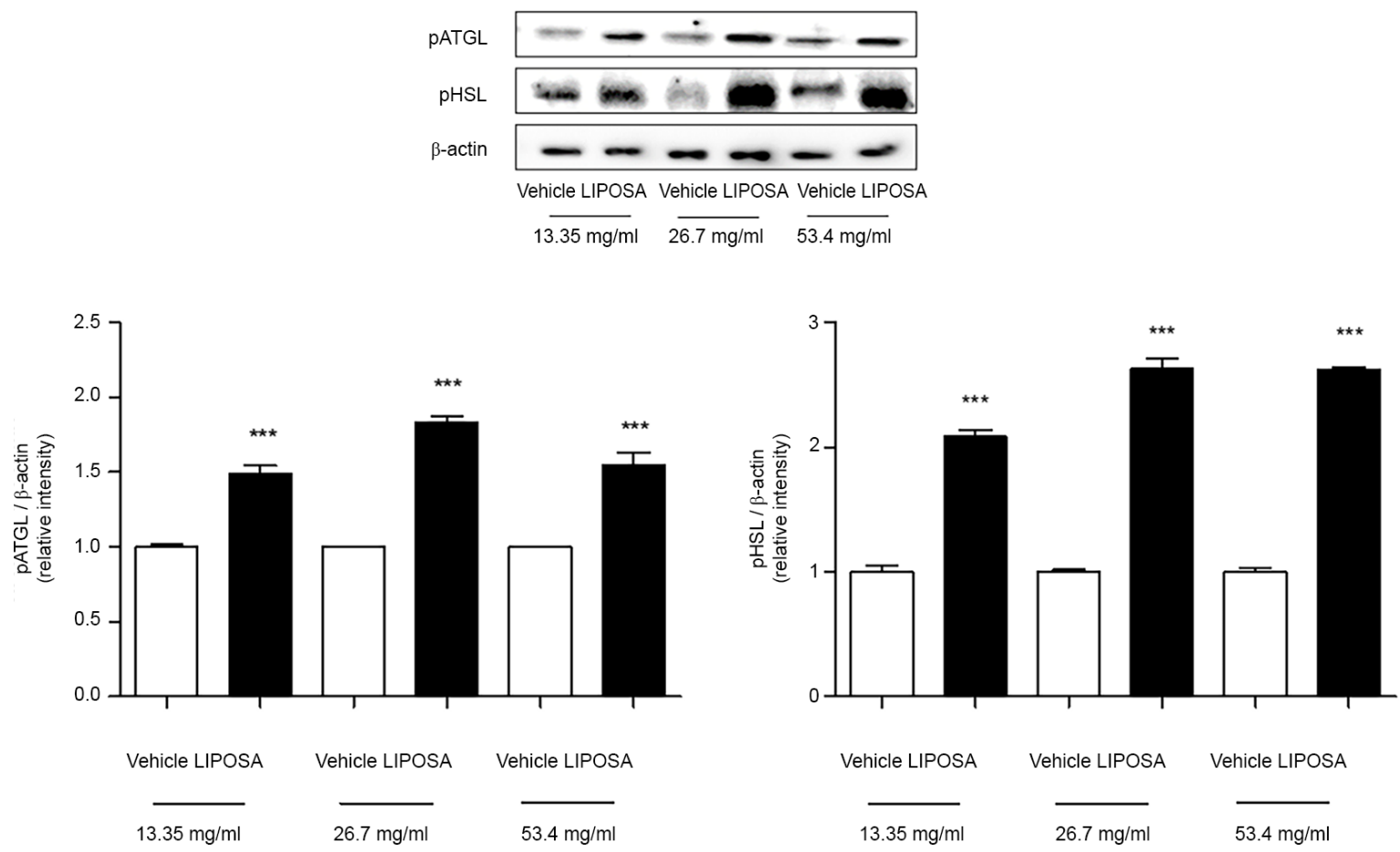

Figure 4. LIPOSA pharmacopuncture elevates the expression levels of lipolytic enzymes in inguinal fat tissues in obese mice. Levels of pATGL/ATGL and pHSL/HSL were analyzed by western blotting. Quantitative data are shown as the mean \pm standard error of the mean. ${ }^{* * *} \mathrm{P}<0.001$ compared with each saline-treated side (vehicle). ATGL, adipose triglyceride lipase; HSL, hormone-sensitive lipase; p, phosphorylated.
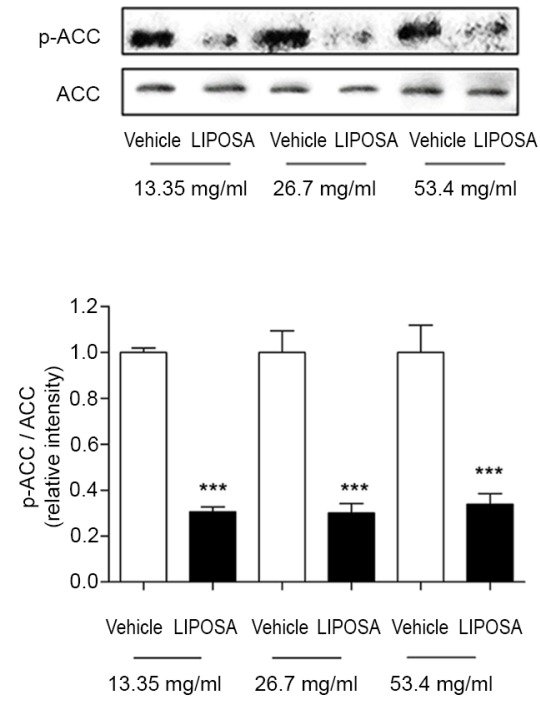
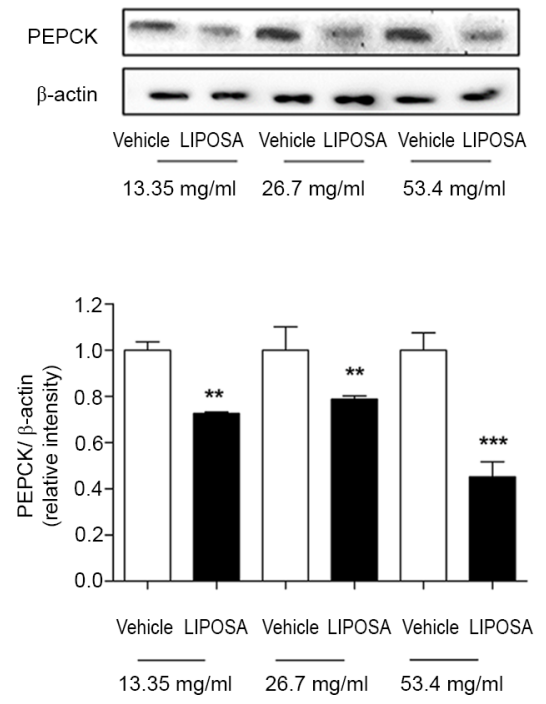
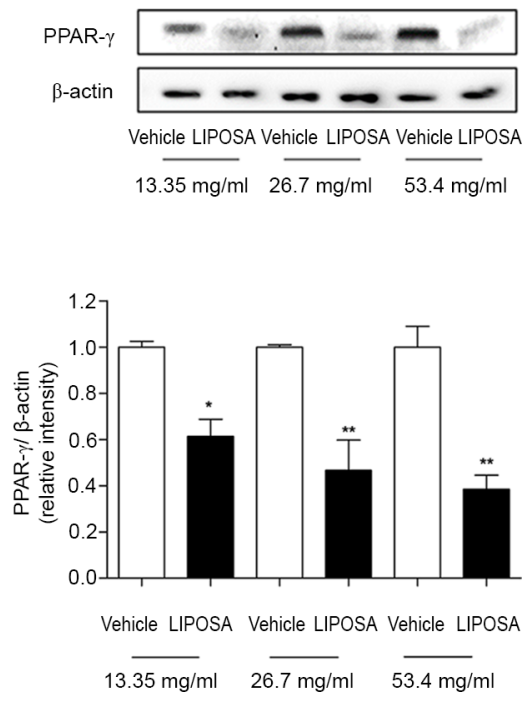

Figure 5. LIPOSA pharmacopuncture decreases the expression levels of ACC, PEPCK and PPAR- $\gamma$ in inguinal fat tissues in obese mice. Expression levels of p-ACC/ACC, PEPCK and PPAR- $\gamma$ were analyzed by western blotting. Quantitative data are shown as the mean \pm standard error of the mean. "P<0.05, ${ }^{* *} \mathrm{P}<0.01$ and ${ }^{* * *} \mathrm{P}<0.001$ compared with each saline-treated side (vehicle). ACC, acetyl-CoA carboxylase; PEPCK, phosphoenolpyruvate carboxykinase; PPAR- $\gamma$, peroxisome proliferator-activated receptors- $\gamma$; p, phosphorylated.

been clearly known (20). Lipolysis by neutral lipases such as ATGL and HSL releases fatty acids and glycerol on triglycerides (21). The triglycerides can be disrupted by lipolytic drugs without an issue of body energy deposition (22). In this study, subcutaneous injection with LIPOSA markedly increased the expressions of lipophagic factors including ATG7, ATG5 and LC3-II, and lipases including ATGL and HSL. Taken together, LIPOSA pharmacopuncture regulated the lipolytic process, especially lipophagy and lipase activation. Based on the results from the fat weight and adipocyte diameter, we expected that LIPOSA pharmacopuncture degraded fat cells containing triglycerides and reduced the enlargement of lipid droplets through lipolysis and lipophagy, which are therapeutic strategies to inhibit localized body fat (Fig. 6).

Apart from lipolysis, molecules were investigated that were involved in lipid accumulation. In addition to the stimulation of lipolysis, strategies to regulate the lipid 
Table I. Changes in concentrations of serum BUN, creatinine, AST and ALT of mice after 2 weeks of LIPOSA pharmacopuncture injection.

\begin{tabular}{|c|c|c|c|c|}
\hline Group & BUN & Creatinine & AST (GOT) & ALT (GPT) \\
\hline Normal mice & $36.67 \pm 0.58$ & $0.26 \pm 0.04$ & $143 \pm 35.16$ & $41.33 \pm 10.26$ \\
\hline $13.35 \mathrm{mg} / \mathrm{ml}$ LIPOSA & $25.80 \pm 2.17$ & $0.27 \pm 0.05$ & $187.50 \pm 40.83$ & $33.00 \pm 7.66$ \\
\hline 26.7 mg/ml LIPOSA & $30.60 \pm 4.67$ & $0.25 \pm 0.04$ & $230.75 \pm 20.56$ & $33.00 \pm 4.97$ \\
\hline $53.4 \mathrm{mg} / \mathrm{ml}$ LIPOSA & $29.00 \pm 2.92$ & $0.29 \pm 0.03$ & $173.25 \pm 44.30$ & $29.00 \pm 5.23$ \\
\hline
\end{tabular}

Data are presented as the mean \pm standard error of the mean. BUN, blood urea nitrogen; ALT, alanine aminotransferase; GOT, glutamate oxaloacetate transaminase; ALT, alanine aminotransferase; GPT, glutamic pyruvate transaminase.

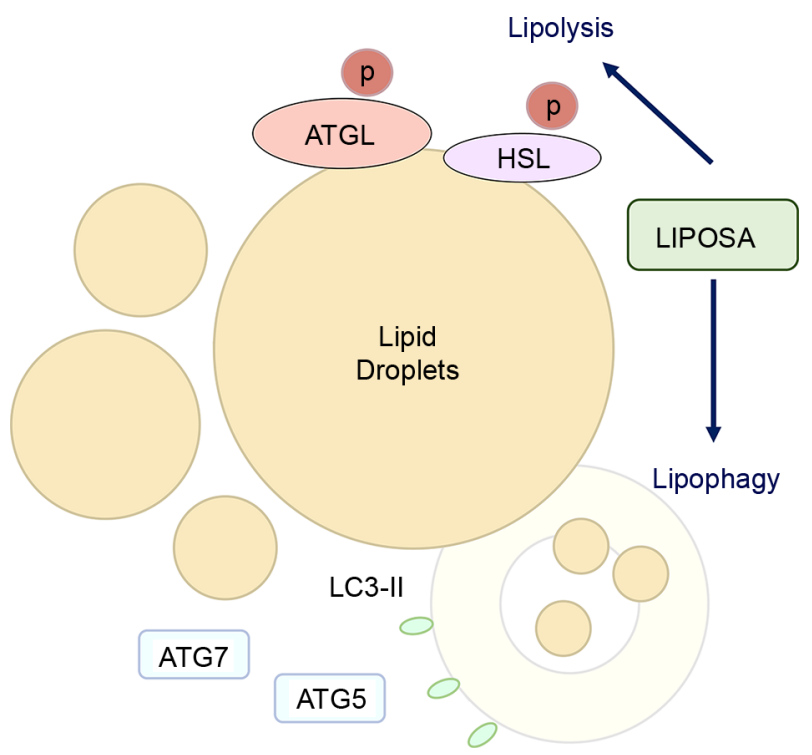

Figure 6. Schematic diagram of the effect of LIPOSA pharmacopuncture on localized fat. LIPOSA injection effectively inhibits the accumulation of localized adiposity by regulating lipophagy and lipolysis-related mechanisms.

metabolism by inhibiting the differentiation of adipocytes (adipogenesis), release of glucose (gluconeogenesis) in adipose tissue or synthesis of fatty acids (lipogenesis) can be targets for obesity and obesity-related diseases (23). The phosphorylation of ACC, a lipogenesis enzyme, has been known to induce the synthesis of fatty acids (24). PPAR- $\gamma$ is highly expressed in white adipose tissues and associated with lipid metabolism (25). Activation of PPAR- $\gamma$ mediates the differentiation of preadipocytes into mature adipocytes (25). In terms of PEPCK, it acts as a regulatory enzyme of gluconeogenesis in adipose tissues (26). An increase in PEPCK activity leads to adipocyte hypertrophy by free fatty acid re-esterification, resulting in fat accumulation (26). LIPOSA pharmacopuncture was found to decrease the expressions of ACC, PPAR- $\gamma$ and PEPCK. Along with the lipolytic effects, LIPOSA pharmacopuncture might inhibit fat accumulation by regulating lipid metabolism.

Taken together, LIPOSA pharmacopunture breaks down localized areas of fat by its lipolytic property. Triglyceride accumulation in the inguinal fat pad was inhibited by LIPOSA subcutaneous injection with its promotive effects on lipophagy and lipase activation. In addition, lipid metabolism related to fat accumulation including adipogenesis, gluconeogenesis and lipogenesis was regulated by the LIPOSA treatment. LIPOSA pharmacopuncture might reduce localized body fat as a lipolytic injection.

\section{Acknowledgements}

Not applicable.

\section{Funding}

The present study was supported by a National Research Foundation of Korea Grant funded by the Korean Government (grant no. NRF-2019R1I1A2A01063598).

\section{Availability of data and materials}

The datasets used and/or analyzed during the current study are available from the corresponding author on reasonable request.

\section{Authors' contributions}

HL, MHK and WMY designed the study and drafted the manuscript. SCJ, LYC and YKN performed the experiments and analyzed the data. MHK and WMY assessed the authenticity of all the raw data. YWM revised and edited the manuscript, supervised the project and obtained the research grants for the current study. All authors read and approved the final manuscript.

\section{Ethics approval and consent to participate}

All animal procedures were approved by the Committee on Care and Use of Laboratory Animals of the Kyung Hee University [approval no. KHUASP(SE)-18-070; Seoul, South Korea].

\section{Patient consent for publication}

Not applicable.

\section{Competing interests}

The authors declare that they have no competing interests. 


\section{References}

1. Sarwer DB, Wadden TA and Foster GD: Assessment of body image dissatisfaction in obese women: Specificity, severity, and clinical significance. J Consult Clin Psychol 66: 651-654, 1998.

2. Moya AP and Sharma D: A modified technique combining vertical and high lateral incisions for abdominal-to-hip contouring following massive weight loss in persistently obese patients. J Plast Reconstr Aesthet Surg 62: 56-64, 2009.

3. Black DW, Shaw M, McCormick B and Allen J: Pathological gambling: Relationship to obesity, self-reported chronic medical conditions, poor lifestyle choices, and impaired quality of life. Compr Psychiatry 54: 97-104, 2013.

4. Friedmann DP, Avram MM, Cohen SR, Duncan DI, Goldman MP, Weiss ET and Young VL: An evaluation of the patient population for aesthetic treatments targeting abdominal subcutaneous adipose tissue. J Cosmet Dermatol 13: 119-124, 2014.

5. Berry MG and Davies D: Liposuction: A review of principles and techniques. J Plast Reconstr Aesthet Surg 64: 985-992, 2011

6. Reeds DN, Mohammed BS, Klein S, Boswell CB and Young VL: Metabolic and structural effects of phosphatidylcholine and deoxycholate injections on subcutaneous fat: A randomized, controlled trial. Aesthet Surg J 33: 400-408, 2013.

7. Noh Y and Heo CY: The effect of phosphatidylcholine and deoxycholate compound injections to the localized adipose tissue: An experimental study with a murine model. Arch Plast Surg 39: 452-456, 2012.

8. Kim SY, Shin IS and Park YJ: Effect of acupuncture and intervention types on weight loss: A systematic review and meta-analysis. Obes Rev 19: 1585-1596, 2018.

9. Huang YC, Tsay HJ, Lu MK, Lin CH, Yeh CW, Liu HK and Shiao YJ: Astragalus membranaceus-polysaccharides ameliorates obesity, hepatic steatosis, neuroinflammation and cognition impairment without affecting amyloid deposition in metabolically stressed APPswe/PS1dE9 mice. Int J Mol Sci 18 : 2746, 2017.

10. Kim YJ, Shin YO, Ha YW, Lee S, Oh JK and Kim YS: Anti-obesity effect of Pinellia ternata extract in Zucker rats. Biol Pharm Bull 29: 1278-1281, 2006.

11. Yang HY and Lee SG: Effects of dandelion (Teraxacum platycarpum) with various extracting method on antioxidative capacity, lipid metabolism in diet-induced obese rats. Korean J Orient Physiol Pathol 25: 48-54, 2011 (In Korean).

12. Heo J: Dongeuibogam. 1613.

13. Pinto H: Local fat treatments: Classification proposal. Adipocyte 5: 22-26, 2016.
14. Bray GA, Fruhbeck G, Ryan DH and Wilding JP: Management of obesity. Lancet 387: 1947-1956, 2016.

15. Engin A: Fat cell and fatty acid turnover in obesity. Adv Exp Med Biol 960: 135-160, 2017

16. Haczeyni F, Bell-Anderson KS and Farrell GC: Causes and mechanisms of adipocyte enlargement and adipose expansion. Obes Rev 19: 406-420, 2018.

17. Lee HJ, Lee MH, Lee SG, Yeo UC and Chang SE: Evaluation of a novel device, high-intensity focused ultrasound with a contact cooling for subcutaneous fat reduction. Lasers Surg Med 48: 878-886, 2016.

18. Cingolani F and Czaja MJ: Regulation and functions of autophagic lipolysis. Trends Endocrinol Metab 27: 696-705, 2016.

19. Singh R and Cuervo AM: Lipophagy: Connecting autophagy and lipid metabolism. Int J Cell Biol 2012: 282041, 2012.

20. Martinez-Lopez N, Garcia-Macia M, Sahu S, Athonvarangkul D, Liebling E, Merlo P, Cecconi F, Schwartz GJ and Singh R: Autophagy in the CNS and periphery coordinate lipophagy and lipolysis in the brown adipose tissue and liver. Cell Metab 23: 113-127, 2016.

21. Kim JH, Kim OK, Yoon HG, Park J, You Y, Kim K, Lee YH, Choi KC, Lee J and Jun W: Anti-obesity effect of extract from fermented Curcuma longa $\mathrm{L}$. through regulation of adipogenesis and lipolysis pathway in high-fat diet-induced obese rats. Food Nutr Res 60: 30428, 2016.

22. Ryden M and Arner P: Subcutaneous adipocyte lipolysis contributes to circulating lipid levels. Arterioscler Thromb Vasc Biol 37: $1782-1787,2017$

23. Kojta I, Chacinska M and Blachnio-Zabielska A: Obesity, bioactive lipids, and adipose tissue inflammation in insulin resistance. Nutrients 12: 1305, 2020.

24. Janovska A, Hatzinikolas G, Staikopoulos V, McInerney J, Mano M and Wittert GA: AMPK and ACC phosphorylation: Effect of leptin, muscle fibre type and obesity. Mol Cell Endocrinol 284: 1-10, 2008.

25. Marion-Letellier R, Savoye G and Ghosh S: Fatty acids, eicosanoids and PPAR gamma. Eur J Pharmacol 785: 44-49, 2016.

26. Franckhauser S, Muñoz S, Pujol A, Casellas A, Riu E, Otaegui P, Su B and Bosch F: Increased fatty acid re-esterification by PEPCK overexpression in adipose tissue leads to obesity without insulin resistance. Diabetes 51: 624-630, 2002.

(c) (i) () This work is licensed under a Creative Commons CCY NG NO Attribution-NonCommercial-NoDerivatives 4.0 International (CC BY-NC-ND 4.0) License. 